\title{
Structure and properties of ilmenite from first principles
}

\author{
N. C. Wilson, ${ }^{1,2}$ J. Muscat,,${ }^{1} *$ D. Mkhonto, ${ }^{3}$ P. E. Ngoepe, ${ }^{3}$ and N. M. Harrison ${ }^{4,5}$ \\ ${ }^{1}$ CSIRO Minerals, Bayview Avenue, Clayton South, Victoria, 3169, Australia \\ ${ }^{2}$ RMIT University, GPO Box 2476V, Melbourne, Victoria, 3001, Australia \\ ${ }^{3}$ Department of Physics, University of the North, Turfloop, South Africa \\ ${ }^{4}$ Department of Chemistry, Imperial College of Science and Technology, London, SW7 2AY, United Kingdom \\ ${ }^{5}$ CCLRC Daresbury Laboratory, Daresbury, Warrington, WA4 4AD, United Kingdom
}

(Received 4 August 2004; published 14 February 2005)

\begin{abstract}
The electron structure, magnetic, structural, and elastic properties of ilmenite $\left(\mathrm{FeTiO}_{3}\right)$ are computed within a hybrid density functional formalism. The computed properties are found to be very sensitive to the treatment of electronic exchange and correlation; Hartree-Fock and generalized gradient approximation calculations are performed for comparison. Within the hybrid formalism a qualitatively correct description of the ground-state electronic structure is obtained. Predicted geometric and elastic parameters are in close agreement with experiment as is the charge transfer excitation energy. The essential features of this functional are its treatment of the electronic self interaction and its reasonable estimate of the pair correlation energy of the doubly occupied Fe- $d$ orbital.
\end{abstract}

DOI: 10.1103/PhysRevB.71.075202

PACS number(s): 71.20.Nr, 61.50.Ah, 71.15.Mb

\section{INTRODUCTION}

Ilmenite, $\mathrm{FeTiO}_{3}$, is a naturally occuring mineral present in igneous rocks derived from the upper mantle down to depths of some $400 \mathrm{~km}$ and thus pressures of $12-13 \mathrm{GPa} .{ }^{1}$ Ilmenite is an economically important mineral primarily because of its role in the production of titania pigments. Its magnetic properties and those of ilmenite-hematite $\left(\mathrm{Fe}_{2} \mathrm{O}_{3}\right)$ solid solutions are of particular importance in commercial extraction via magnetic separation. The magnetic behavior of the solid solution is also of crucial importance to studies in which rock magnetization is used to interpret historical fluctuations in the Earth's magnetic field as rocks containing it display self-reversed magnetization - that is, the spontaneous magnetization occuring as the rock is cooled below the Curie temperature opposes the applied field. ${ }^{2,3}$

Ilmenite is a wide bandgap semiconductor with a measured band gap of about $2.5 \mathrm{eV}$. ${ }^{4,5} \mathrm{~A}$ useful starting point for the analysis of the electronic structure of transition metal oxides is the ionic model in which formal charges are assigned to the ions. For $\mathrm{FeTiO}_{3}$ this is complicated by the fact that there are two distinct cation charge orderings consistent with $\mathrm{O}^{2-}$ anions, i.e., $\mathrm{Fe}^{3+} \mathrm{Ti}^{4+}$ and $\mathrm{Fe}^{3+} \mathrm{Ti}^{3+}$. The charge transfer excitation between these two states is well known and has been observed and modeled in a number of $\mathrm{Fe}$ and $\mathrm{Ti}$ bearing minerals. ${ }^{6}$ This flexibility of the electronic structure of the Fe cation is extremely difficult to model using simulations based on empirical potentials. The delicate energy balance between the two charge states is difficult to model and simple assignment of a particular charge to a site may lead to incorrect interactions. This is complicated further as the orbital occupancy of the highly localized $d$ orbitals of the transition metal ions is not obvious a priori and both lowspin and high-spin states of the Fe ion are possible. An approach within which the structural, charge ordering, orbital ordering, and magnetic energy scales are computed reliably is required. Quantum mechanical simulations based on den- sity functional theory (DFT) offer a potential solution but these methods also face difficulties. The local electronic configurations of the $\mathrm{Fe}^{2+}$ and $\mathrm{Fe}^{3+}$ states are $d^{6}$ and $d^{5}$, respectively. The correlation energy difference between these states is large and in a pure octahedral environment the $d^{6}$ state is degenerate and thus a strong interaction between orbitaloccupancy and the local structure is to be expected. The widely used local density and generalized gradient approximations (LDA and GGA) to DFT have a strong tendency to describe wide band gap magnetic insulators as nonmagnetic metals due to the incorrect treatment of electronic selfinteraction.

The dependence of ilmenite's structure on temperature, pressure and composition is strongly coupled to its electronic, magnetic, and optical properties. A detailed understanding of this coupling is of fundamental interest in the study of strongly interacting systems and also of great importance to a number of commercial processes. Computer simulation provides an ideal probe to examine the interactions between spin, charge, orbital, and structural degrees of freedom, which are difficult to obtain from experiment alone.

The current work demonstrates that reliable and predictive simulations of the structure and properties of ilmenite can be obtained using a hybrid-exchange density functional approach. This approximation retains, and improves on, the GGA's description of the ground-state energy surface while introducing sufficient self-interaction to allow a reasonable description of the states surrounding the Fermi energy to be obtained. The article is organized as follows. In Sec. II the crystallographic structures of $\mathrm{FeTiO}_{3}$ are described. Section III discusses the details of the calculations. The computed structure, electronic structure, and magnetic properties are presented in Sec. IV and the results are discussed in Sec. V. The main conclusions are summarized in Sec. VI.

\section{DESCRIPTION OF THE STRUCTURES}

Ionic, or semi-ionic, materials with $A B \mathrm{O}_{3}$ composition tend to adopt either the high coordination cubic perovskite 


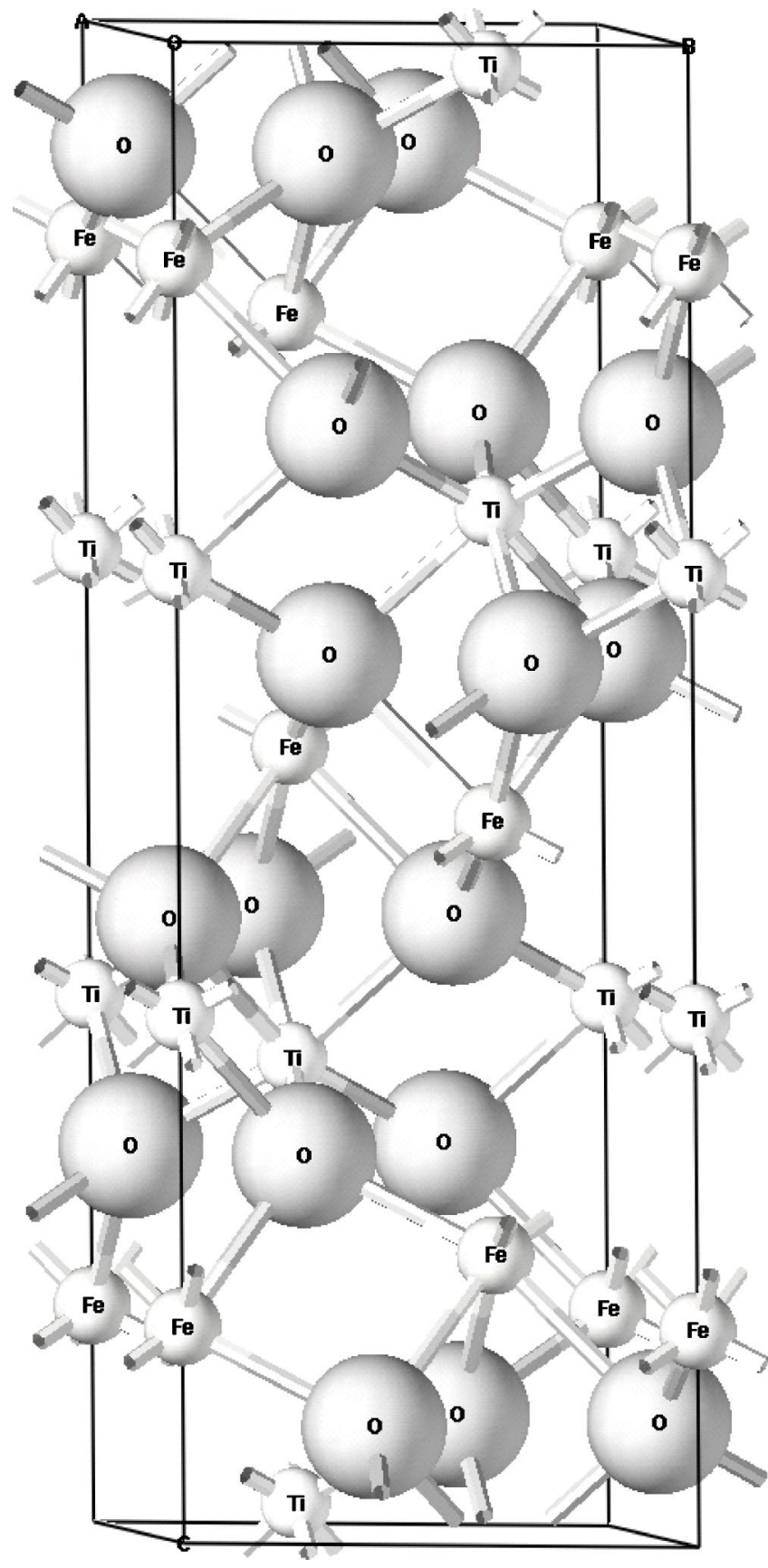

FIG. 1. The structure of $\mathrm{FeTiO}_{3}$.

form (should ionic packing allow) or a lower coordination sesquioxide structure. The two common sesquioxide structures are the ilmenite, which is displayed in Fig. 1, and the lithium niobate structures. Both of these structures are based on the higher symmetry corundum $\left(\mathrm{B}_{2} \mathrm{O}_{3}\right)$ structure (space group $R \overline{3} c$ ) which is based on a hexagonal close-packed anion lattice with cations occupying two thirds of the available octahedral interstices such that $A \mathrm{O}_{6}$ octahedra share edges in a honeycomb arrangement in (001) planes and share octahedral faces between adjacent planes. The metal atoms all lie on threefold axes parallel to c, forming the sequence $A-A-\square-A-A$ of face-shared pairs of octahedra alternating with vacant octahedral sites $(\square)$.

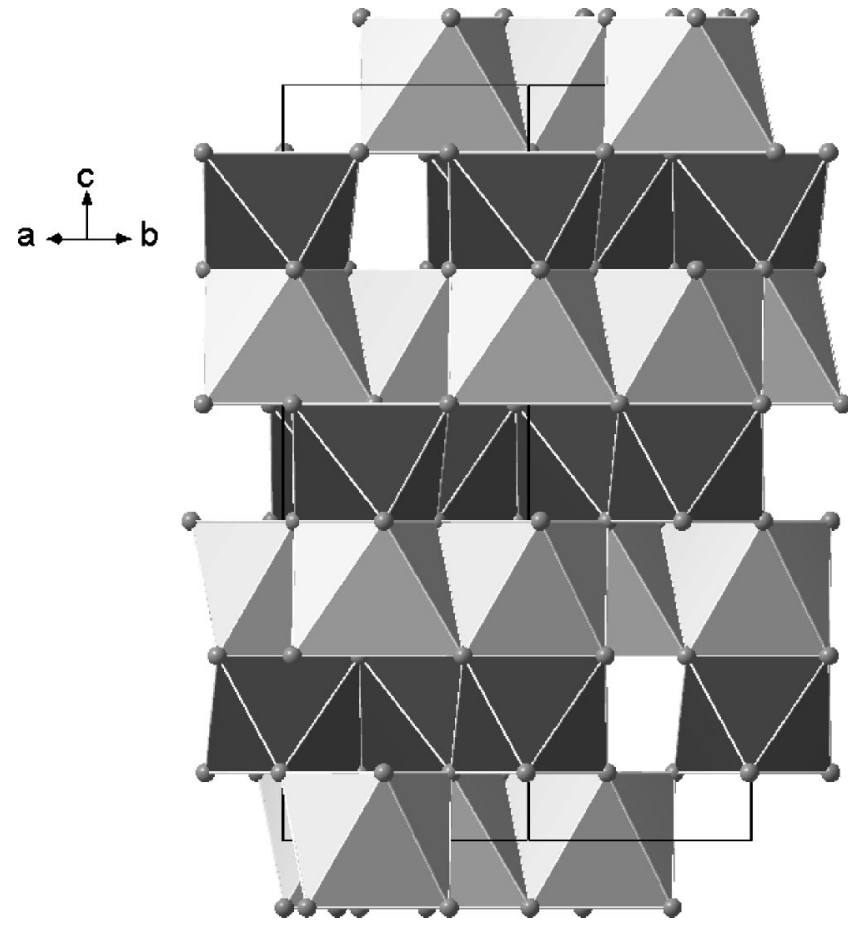

FIG. 2. The $\mathrm{FeTiO}_{3}$ structure showing $\mathrm{FeO}_{6}$ (light color) and $\mathrm{TiO}_{6}$ (dark color) octahedra.

In ilmenite, there are two types of metal ions, which form alternating bilayers of $\mathrm{Fe}$ and $\mathrm{Ti}$ ions perpendicular to the c axis, with $-\mathrm{Fe}-\mathrm{Ti}-\square-\mathrm{Ti}-\mathrm{Fe}-$ ordering along the threefold axes, reducing the symmetry to $R \overline{3}$. The Fe and Ti ions are octahedrally coordinated to $\mathrm{O}$ ions with three octahedral edges shared between cation octahedra of the same type (see Fig. 2). Each cation octahedron shares one octahedral face with a cation of the other type in an adjacent bilayer whereas the face opposite is shared with an empty octahedral position ("hole"). The crystallographic cell contains two formula units of $\mathrm{FeTiO}_{3}$ with $\mathrm{Fe}$ ions at $\pm\left(0,0, \mathrm{Fe}_{z}\right)$, Ti ions at $\pm\left(0,0, \mathrm{Ti}_{z}\right)$ and oxygen ions at $\pm\left(\mathrm{O}_{x}, \mathrm{O}_{y}, \mathrm{O}_{z} ;-\mathrm{O}_{y}, \mathrm{O}_{x}-\mathrm{O}_{y}, \mathrm{O}_{z} ; \mathrm{O}_{y}-\mathrm{O}_{x},-\mathrm{O}_{x}, \mathrm{O}_{z}\right)$. The lithium niobate form differs by having mixed $\mathrm{Fe}$, Ti bilayers, and a threefold axis ordering of $-\mathrm{Fe}-\mathrm{Ti}-\square-\mathrm{Fe}-\mathrm{Ti}-$.

$\mathrm{FeTiO}_{3}$ adopts the ilmenite, lithium niobate, or perovskite structures depending on the temperature and pressure, with ilmenite being the stable phase at ambient pressure. The pressure of transition from ilmenite to the perovskite is $16 \mathrm{GPa}$ at room temperature, with the lithium niobate phase being a quench phase from the perovskite structure. The lithium niobate structure transforms reversibly to perovskite at $16 \pm 1 \mathrm{GPa}$ at room temperature. ${ }^{7,8}$

\section{DETAILS OF THE CALCULATIONS}

All calculations were performed using the CRYSTAL software package, ${ }^{9}$ which is based on the periodic, all-electron, linear combination of atomic orbitals (LCAO) formalism where the Bloch orbitals of the crystals are expanded using atom centered Gaussians orbitals with $s, p$, or $d$ symmetry. The most significant numerical approximation made in the 
calculations is based on the choice of the local Gaussian basis sets. The basis sets used here were developed and optimised in previous calculations on rutile $\mathrm{TiO}_{2}$ and bulk surfaces ${ }^{10-14}$ and studies of hematite $\mathrm{Fe}_{2} \mathrm{O}_{3} \cdot{ }^{15}$ The general quality of the basis set is triple valence; that is, three independent functions are used to expand the valence electrons of each ionic type. In addition $d$-symmetry polarization functions were introduced on the oxygen ions. Full details of the basis sets are available on the internet. ${ }^{16}$

The Coulomb and exchange series are evaluated in direct space and truncated at long range using Gaussian overlap criteria. The details of this procedure have been described elsewhere. ${ }^{9,17}$ The criteria are determined by five cutoff tolerances which in the current study were set to $10^{-7}, 10^{-7}$, $10^{-7}, 10^{-7}$, and $10^{-14}$ which typically converge total energy differences between related structures to within $0.1 \mathrm{mHa}$. The reciprocal space integration was performed by sampling the Brillouin zone using the Monkhorst-Pack ${ }^{9,18}$ scheme with a shrinking factor of 4 . This gives 14 symmetry nonequivalent points in the irreducible Brillouin zones of the bulk crystals.

The two most common treatments of electronic exchange and correlation in insulators are Hartree-Fock (HF) and density functional theory (DFT). HF theory treats exchange exactly as a nonlocal, orbital dependent, potential within the single determinant approximation but neglects correlation effects. Within DFT both exchange and correlation are approximated as local potentials typically by means of either the local density (LDA) or generalized gradient (GGA) approximations. An alternate approach is to use a hybrid theory has which combines elements of both HF and DFT. The most commonly used hybrid theory implementation is a modified form of Becke's three parameter hybrid functional ${ }^{19}$ (B3LYP) as employed by Stephens et al. ${ }^{20} \mathrm{Here}$, the exchange and correlation is given by

$$
\begin{aligned}
E_{\mathrm{XC}}= & \left(1-a_{0}\right) E_{X}^{\mathrm{LDA}}+a_{0} E_{X}^{\mathrm{HF}}+a_{x} \Delta E_{X}^{\mathrm{B} 88}+a_{c} E_{C}^{\mathrm{LYP}} \\
& +\left(1-a_{c}\right) E_{C}^{\mathrm{VWN}},
\end{aligned}
$$

where $a_{x} \Delta E_{X}^{\mathrm{B} 88}$ is Becke's gradient correction to the exchange functional, ${ }^{21}$ and the correlation is given by a combination of the Lee-Yang-Parr ${ }^{22}$ (LYP) and the Vosko-Wilk-Nussair ${ }^{23}$ functionals. Here, Becke's original three empirical parameters $\left(a_{0}=0.2, a_{x}=0.72\right.$, and $\left.a_{c}=0.81\right)$ are used. These parameters were determined by optimization with respect to the observed atomization energies, ionization potentials and proton affinities of a number of small molecules. ${ }^{21}$ In addition to a significant improvement in the description of the ground-state energetics, ${ }^{24}$ solid state calculations using this functional have been shown to yield band structures ${ }^{25}$ structural properties, ${ }^{26}$ and magnetic couplings ${ }^{27}$ in as good as or better agreement with observation than achieved using GGA functionals. Calculations were performed using the spin unrestricted $\mathrm{HF}$ and B3LYP theories.

The lattice parameters and internal coordinates of $\mathrm{FeTiO}_{3}$ were optimised using a modified Broyden-FletcherGoldfarb-Shanno (BFGS) algorithm to a tolerance of $0.0005 \AA$ in the structure and $10^{-7} \mathrm{Ha}$ in the total energy. Calculations at pressures other than $0 \mathrm{GPa}$ were performed

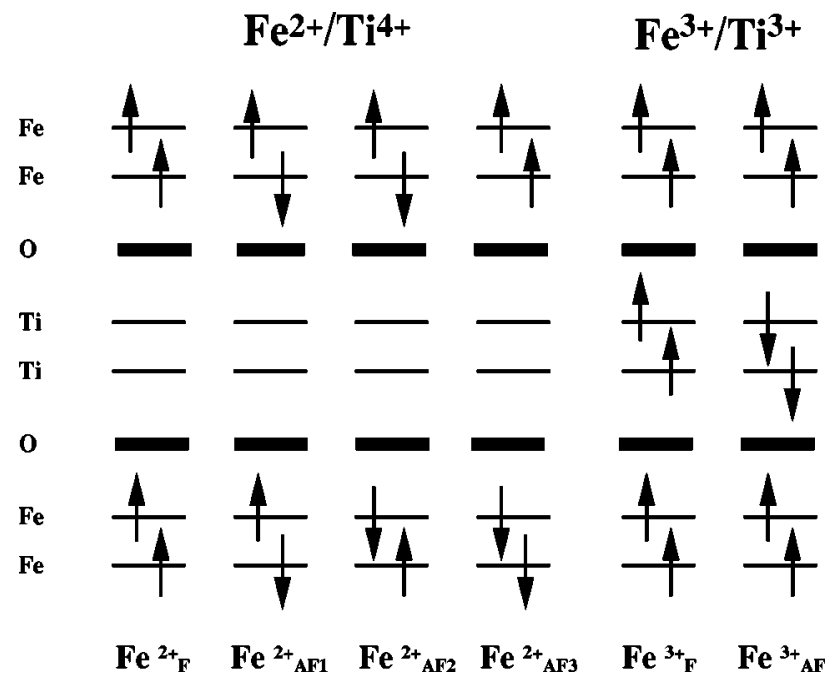

FIG. 3. The various spin arrangements considered for the $\mathrm{Fe}^{2+} / \mathrm{Ti}^{4+}$ and $\mathrm{Fe}^{3+} / \mathrm{Ti}^{3+}$ states.

by applying a hydrostatic pressure and performing unconstrained minimization of the enthalpy with respect to both internal coordinates and cell parameters. The bulk modulus was computed by fitting the results from a set of optimizations at nine pressure values between -2 and $10 \mathrm{GPa}$ to a Murnaghan equation of state $^{28}$ of the form

$$
U(V)=\frac{B_{0} V}{B_{0}^{\prime}}\left[\frac{\left(\frac{V_{0}}{V}\right)^{B_{0}^{\prime}}}{B_{0}^{\prime}-1}+1\right]+U_{0},
$$

where $U(V)$ is the total internal energy, $V$ the unit cell volume, $V_{0}$ the equilibrium volume, $B_{0}$ the bulk modulus with pressure derivative $B_{0}^{\prime}$, and $U_{0}$ is the equilibrium total energy.

\section{RESULTS}

\section{A. Electronic and magnetic structure}

Initial calculations were performed at the experimental geometry. ${ }^{29}$ The self-consistent field (SCF) calculations are initiated with a density matrix formed from a superposition of atomic or ionic charge densities. In strongly localized systems it is possible to converge to a number of different fixed points of the SCF procedure by variation of the initial conditions. The lowest-energy state is the ground state for a particular geometry while the other fixed points correspond to possible excited states. To achieve the various charge and spin states, initial orbital occupations and atomic spins were modified to resemble those of the desired state and, if needed, the net spin of the system was constrained for the first few cycles of the SCF procedure. In this way solutions corresponding to the $\mathrm{Fe}^{2+} / \mathrm{Ti}^{4+}$ and $\mathrm{Fe}^{3+} / \mathrm{Ti}^{3+}$ states were converged within both the HF and B3LYP approximations. The solutions for these charge states with ferromagnetic coupling are denoted here as $\mathrm{Fe}_{F}^{2+}$ and $\mathrm{Fe}_{F}^{3+}$, respectively. The various spin arrangements considered below, and the notation used to refer to them herein, are illustrated in Fig. 3. 
TABLE I. Energy differences (relative to lowest energy state per $\mathrm{FeTiO}_{3}$ formula unit in eV) and Mulliken population analysis of net charge $(\alpha+\beta)$ and net spin $(\alpha-\beta)$ for ilmenite at $0 \mathrm{GPa}$, computed using B3LYP and HF theory. The state labels are defined in the text and illustratted in Fig. 3.

\begin{tabular}{lccccccc}
\hline \hline & & \multicolumn{3}{c}{ Net Charge } & \multicolumn{3}{c}{ Net spin } \\
\cline { 3 - 9 } State & energy (eV) & $\mathrm{Fe}$ & $\mathrm{Ti}$ & $\mathrm{O}$ & $\mathrm{Fe}$ & $\mathrm{Ti}$ & $\mathrm{O}$ \\
\hline & & \multicolumn{3}{c}{$\mathrm{B} 3 \mathrm{LYP}$} \\
$\mathrm{Fe}_{F}^{2+}$ & 0.0000 & 24.26 & 19.68 & 9.36 & 3.76 & 0.01 & 0.08 \\
$\mathrm{Fe}_{\mathrm{LS}}^{2+}$ & 1.5199 & 24.52 & 19.67 & 9.27 & 0.00 & 0.00 & 0.00 \\
$\mathrm{Fe}_{F}^{3+}$ & 2.5366 & 23.90 & 19.90 & 9.40 & 4.26 & 0.94 & 0.27 \\
& & & & & & & \\
$\mathrm{Fe}_{F}^{2+}$ & 0.0000 & 24.17 & 19.13 & 9.57 & 3.90 & 0.01 & 0.03 \\
$\mathrm{Fe}_{\mathrm{LS}}^{2+}$ & 3.4512 & 24.26 & 19.13 & 9.54 & 0.00 & 0.00 & 0.00 \\
$\mathrm{Fe}_{F}^{2+}$ & 0.7808 & 23.46 & 19.56 & 9.66 & 4.74 & 0.98 & 0.09 \\
\hline \hline
\end{tabular}

The differences in the computed total energies for these charge states in high-spin and low-spin configurations are summarised in Table I. Both within the HF and B3LYP approximations the $\mathrm{Fe}_{F}^{2+}$ state is found to be the most stable. However, the relative stabilities of the charge states depends significantly on the treatment of exchange and correlation. Within B3LYP the $\mathrm{Fe}_{F}^{3+}$ state is $2.54 \mathrm{eV}$ above the ground state while within $\mathrm{HF}$ is $0.78 \mathrm{eV}$ higher. The solution with $\mathrm{Fe}$ in the diamagnetic, low-spin configuration $\left(\mathrm{Fe}_{\mathrm{LS}}^{2+}\right)$ is $1.52 \mathrm{eV}$ above the ground state within B3LYP and $3.45 \mathrm{eV}$ higher within HF. The charge ordering and on-site spin coupling energies are very sensitive to the treatment of exchange and correlation.

Some insight into the nature of the various solutions can be gained from Mulliken population analysis of the charge and spin densities. The overall picture of the charge and spin distribution amongst the ions is qualitatively similar within the HF and B3LYP approximaitons. For example, the charge (spin) for an $\mathrm{Fe}^{2+}$ ion is $2.43|e|(3.8|e|)$ within B3LYP and $24.2|e|(3.9|e|)$ within HF. Both solutions are consistent with a simple ionic picture of a high-spin $d^{6} \mathrm{Fe}^{2+}$ ion. The electronic state can be analyzed further in terms of the occupation and spin of the individual orbitals in the $d$ shell. The occupations and spins computed with the B3LYP approxima- tion for both the $\mathrm{Fe}_{F}^{2+}$ and $\mathrm{Fe}_{F}^{3+}$ states are presented in Table II. Within the ionic model the $\mathrm{Fe} d^{6}$ state in $\mathrm{Fe}_{F}^{2+}$ state is expected to have four singly occupied and one doubly occupied $d$ orbital. Table II confirms this simple picture with the $d z^{2}$ orbital essentially doubly occupied (an occupancy of $1.8|e|$ and a net spin of about $0|e|$ ). The other $d$ orbitals are singly occupied (with a spin of $1|e|$ ). It is, of course, possibly that other stable states exist in which different $d$ orbitals are doubly occupied. Such states have been converged and found to be metastable with energies around $4 \mathrm{eV}$ above the ground state and typically with metallic character. Supplementary calculations performed using GGA functionals were found to yield only metallic solutions without strong localization into a particular $d$ occupancy supporting the assertion made in Sec. I that the treatment of electronic self-interaction is essential.

For the $\mathrm{Fe}_{F}^{3+}$ state, within the B3LYP approximation, the extra electron on the Ti site localizes in the Ti $3 d$ shell in a $d z^{2}$ orbital yielding a spin on the Ti ion of about $0.8|e|$. On the Fe $d^{5}$ ion all the orbitals are singly occupied (and hence each have a spin of about $1|e|)$. Again this electronic structure of this state is remarkably consistent with a simply ionic model.

In addition to the ferromagnetically coupled state discussed thus far there are a number of possible spin orderings the most likely of which are displayed in Fig. 3. In the $\mathrm{Fe}_{\mathrm{AF} 1}^{2+}$ state the Fe spins are antiferromagnetically aligned both within a bilayer and between bilayers, in the $\mathrm{Fe}_{\mathrm{AF} 2}^{2+}$ state the $\mathrm{Fe}$ spins are antiferromagnetically aligned within a bilayer but ferromagnetically between bilayers and in $\mathrm{Fe}_{\mathrm{AF} 3}^{2+}$ the $\mathrm{Fe}$ spins are ferromagnetically aligned with a bilayer but antiferromagnetically aligned between bilayers. Due to the different spin symmetries each of these arrangements can be converged as a metastable state within supercells containing up to four $\mathrm{FeTiO}_{3}$ formula units. The relative energies of these states for both charge configurations are presented in Table III. The energy scale for magnetic ordering is about two orders of magnitude smaller than that for charge ordering.

The $\mathrm{Fe}_{\mathrm{AF} 3}^{2+}$ state is the experimentally observed spin ordering below the Néel temperature of $57.35 \mathrm{~K} .{ }^{30}$ Within the B3LYP approximation the $\mathrm{Fe}_{\mathrm{AF} 1}^{2+}$ and $\mathrm{Fe}_{\mathrm{AF} 2}^{2+}$ arrangements are the lowest-energy configurations. The observed $\mathrm{Fe}_{\mathrm{AF} 3}^{2+}$ state is $0.02 \mathrm{eV}$ higher in energy. Within the HF approximation the $\mathrm{Fe}_{\mathrm{AF} 3}^{2+}$ arrangement is found to be the most stable but is es-

TABLE II. Mulliken population analysis of net charge $(\alpha+\beta)$ and net spin $(\alpha-\beta)$ for the cations in the $\mathrm{Fe}_{F}^{2+}$ and $\mathrm{Fe}_{F}^{3+}$ states as computed using B3LYP at the experimental geometry.

\begin{tabular}{lcccccccc}
\hline \hline & \multicolumn{3}{c}{$\mathrm{Fe}_{F}^{2+}$} \\
\cline { 2 - 10 } & \multicolumn{3}{c}{$\mathrm{Fe}$} & \multicolumn{2}{c}{$\mathrm{Ti}$} & \multicolumn{3}{c}{$\mathrm{Fe}_{F}^{3+}$} \\
\hline Orbital & Occ & Spin & Occ & Spin & Occ & Spin & Occ & Spin \\
$d z^{2}$ & 1.84 & 0.14 & 0.22 & -0.01 & 1.10 & 0.90 & 0.83 & 0.74 \\
$d x z$ & 1.09 & 0.90 & 0.34 & 0.00 & 1.20 & 0.81 & 0.26 & 0.05 \\
$d y z$ & 1.09 & 0.90 & 0.34 & 0.00 & 1.20 & 0.81 & 0.26 & 0.05 \\
$d x^{2}-y^{2}$ & 1.08 & 0.90 & 0.31 & 0.01 & 1.14 & 0.86 & 0.23 & 0.05 \\
$d x y$ & 1.08 & 0.90 & 0.31 & 0.01 & 1.14 & 0.86 & 0.23 & 0.05 \\
\hline \hline
\end{tabular}


TABLE III. The relative energetics (in $\mathrm{eV}$, per $\mathrm{FeTiO}_{3}$ formula unit) of the ferromagnetic and antiferromagnetic configurations of ilmenite. Labels refer to Fig. 3.

\begin{tabular}{|c|c|c|}
\hline \multirow[b]{2}{*}{ State } & \multicolumn{2}{|c|}{ Energy relative to the $F$ state, $\mathrm{eV}$} \\
\hline & B3LYP & $\mathrm{HF}$ \\
\hline & \multicolumn{2}{|c|}{$\mathrm{Fe}^{2+} / \mathrm{Ti}^{4+}$} \\
\hline $\mathrm{Fe}_{F}^{2+}$ & 0.0000 & 0.0000 \\
\hline $\mathrm{Fe}_{\mathrm{AF} 1}^{2+}$ & -0.0302 & 0.0046 \\
\hline $\mathrm{Fe}_{\mathrm{AF} 2}^{2+}$ & -0.0287 & 0.0047 \\
\hline \multirow[t]{2}{*}{$\mathrm{Fe}_{\mathrm{AF} 3}^{2+}$} & -0.0108 & 0.0000 \\
\hline & \multicolumn{2}{|c|}{$\mathrm{Fe}^{3+} / \mathrm{Ti}^{3+}$} \\
\hline $\mathrm{Fe}_{F}^{3+}$ & 0.0000 & 0.0000 \\
\hline FeAF3+ & & 0.0340 \\
\hline
\end{tabular}

sentially degenerate with $\mathrm{Fe}_{F}^{2+}$. The $\mathrm{Fe}_{\mathrm{AF} 1}^{2+}$ and $\mathrm{Fe}_{\mathrm{AF} 2}^{2+}$ are $0.005 \mathrm{eV}$ higher in energy. The magnetic coupling is extremely sensitive to the treatment of exchange and correlation, with the B3LYP approximation antiferromagnetic and the HF favoring ferromagnetic coupling within the bilayers. In addition the coupling energy is one order of magnitude smaller in the HF approximation. The interbilayer coupling which is mediated by the Ti bilayer is very small.

In the $\mathrm{Fe}^{3+} / \mathrm{Ti}^{3+}$ state, the spin on the $\mathrm{Ti}^{3+}$ ion can align either ferromagnetic $\left(\mathrm{Fe}_{F}^{3+}\right)$ or antiferromagnetically $\left(\mathrm{Fe}_{\mathrm{AF}}^{3+}\right)$ with the spin on the Fe ions (see Fig. 3). Within the B3LYP approximation, the $\mathrm{Fe}_{\mathrm{AF}}^{3+}$ state is unstable and reverts to the $\mathrm{Fe}_{F}^{2+}$ state. However, both states are stable within the HF approximation and the $\mathrm{Fe}_{F}^{3+}$ state is favored by $0.03 \mathrm{eV}$. Within HF the coupling between the $\mathrm{Fe}$ and $\mathrm{Ti}$ bilayers is thus ferromagnetic in nature and significantly larger than the coupling within the $\mathrm{Fe}$ bilayers in the $\mathrm{Fe}^{2+} / \mathrm{Ti}^{4+}$ state.

The charge transfer excitation energy between the $\mathrm{Fe}^{2+}$ and $\mathrm{Ti}^{4+}$ has been measured using optical absorption experiments to be $2.15 \mathrm{eV}\left(20200 \mathrm{~cm}^{-1}\right) .{ }^{31}$ The predicted excitation energy within the B3LYP approximation is in close correspondence with this measurement, $2.54 \mathrm{eV}\left(20459 \mathrm{~cm}^{-1}\right)$, while the HF estimate is much lower, $0.78 \mathrm{eV}$.

The electronic structure and nature of the bonding can be examined further using Mulliken projected densities of states (DOS). The DOS computed for the $\mathrm{Fe}_{F}^{2+}$ and $\mathrm{Fe}_{F}^{3+}$ states within both the HF and B3LYP approxiations are presented in Figs. 4 and 5, respectively. For the $\mathrm{Fe}_{F}^{2+}$ state both $\mathrm{HF}$ and B3LYP approximations predict the material to be an insulator and yield a qualitatively similar picture of the DOS. The valence band is composed primarily of a mixture of $\mathrm{O} 2 p$ and $\mathrm{Fe} 3 d$ states with a small contribution from the $\mathrm{Ti} 3 d$ states. There is significantly more hybridization of the $\mathrm{Fe} 3 d$ and $\mathrm{O} 2 p$ states within the B3LYP approximation which is consistent with the overall greater ionic character of the HF solution noted above.

The detailed structure of the Fe $3 d$ states is very different in the two approximations. Within HF the four singly occupied orbitals ( $\alpha$ spin orbitals) contribute strongly to the peak between -7 and $-9 \mathrm{eV}$, the $\alpha$ and $\beta$ orbitals of the doubly occupied $d z^{2}$ orbital are strongly split from these states to

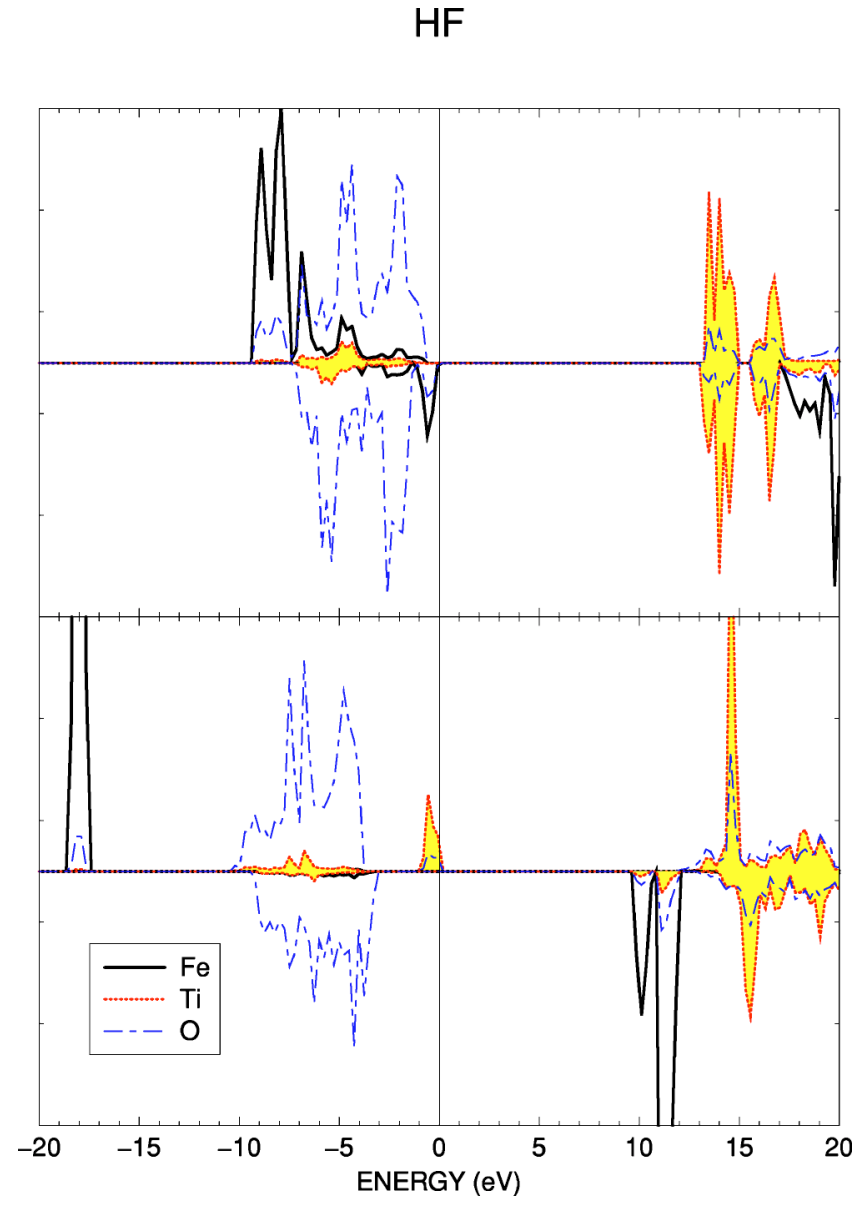

FIG. 4. (Color online) The density of states of ilmenite computed within the HF approximation at the observed geometry. That for the $\mathrm{Fe}_{F}^{2+}$ state is displayed in the top panel and for $\mathrm{Fe}_{F}^{3+}$ bottom panel.

higher energies. The $\alpha$ state forms the peak between -6 and $-7 \mathrm{eV}$ while the $\beta$ state is split from the top of the valence band between 0 and $-1 \mathrm{eV}$. This structure is as one would expect from the ionic model. The doubly occupied $d z^{2}$ states are both pushed to higher energies by their mutual Coulomb repulsion but are split because the $\alpha$ state is stabilized somewhat by on-site exchange interaction with the four other occupied $\alpha$ orbitals.

The B3LYP solution yields a valence band where the Fe $3 d \alpha$ states are strongly hybridized with the $\mathrm{O} 2 p$ valence band. The $\alpha$-spin $d z^{2}$ state contributes strongly to the top of the valence band while the $\beta$-spin $d z^{2}$ state is split with sites almost $2 \mathrm{eV}$ above the valence band maximum. The on-site energy scales expected from the ionic model are clear in the overall structure of Fe $d$ states but are strongly modified by hybridiation with $\mathrm{O} 2 p$ states.

The overall valence band width varies from 7 (B3LYP) to $9 \mathrm{eV}(\mathrm{HF})$. The nature of the band gap is also different in the two formalisms; the lower conduction band edge is predominantly of $\mathrm{Ti} 3 d$ character in the $\mathrm{HF}$ solution and of $\mathrm{Fe} 3 d$ character in the B3LYP solution.

It is well know that HF eigenvalues considerably overestimate occupied band widths and band gaps while recent studies have shown B3LYP eigenvalues reproduce band gaps 


\section{B3LYP}

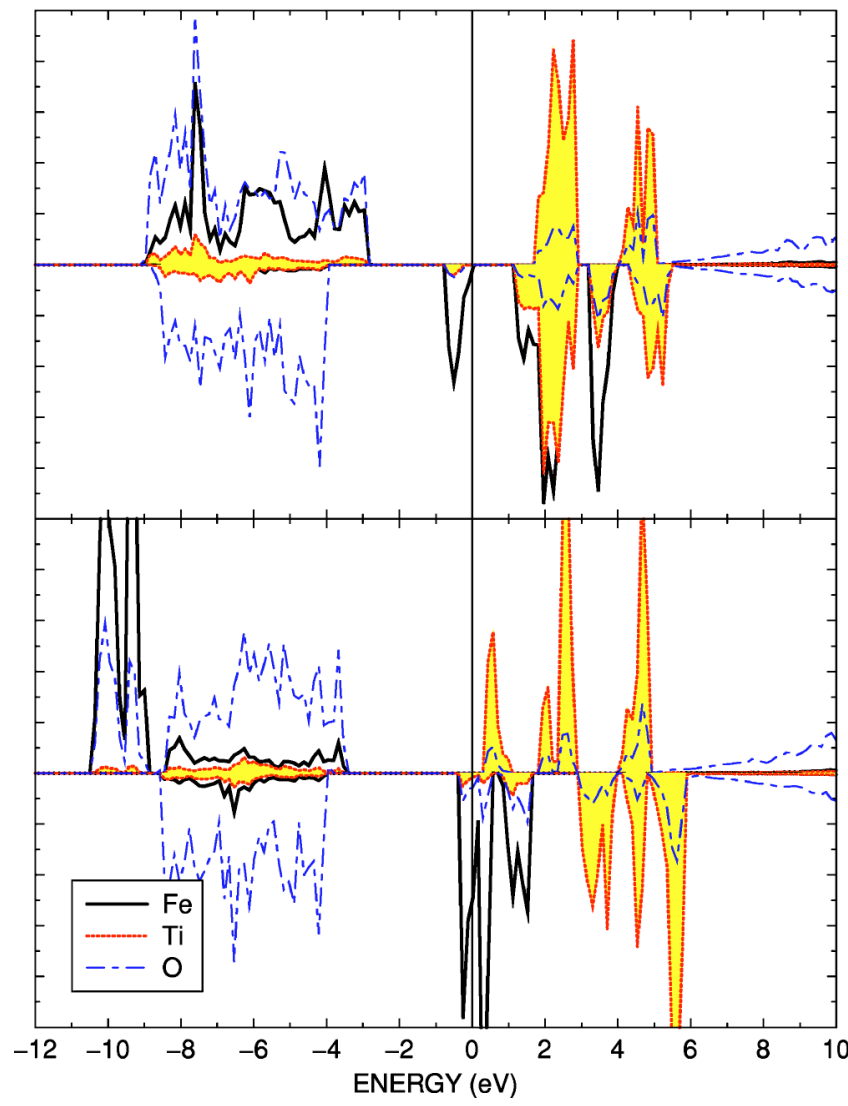

FIG. 5. (Color online) The density of states of ilmenite computed within the B3LYP approximation at the optimized geometry. That for the $\mathrm{Fe}_{F}^{2+}$ state is displayed in the top panel and for $\mathrm{Fe}_{F}^{3+}$ bottom panel.

rather well (within 10\%) for a wide range of materials. ${ }^{25} \mathrm{In}$ the current case, the HF approximation yields a gap of $13 \mathrm{eV}$ and B3LYP a gap of $1.2 \mathrm{eV}$.

Electrochemical measurements of the band gap, give values of between 2.58 and $2.9 \mathrm{eV} ., 32,33$ Diffuse reflectance spectra of ilmenite show absorption peaks at energies of about $1,2.5$, and $4 \mathrm{eV},{ }^{31}$ however do not provide definitive measurements of band gaps. Strens and Wood $^{31}$ attribute the $1 \mathrm{eV}$ transition to a 2 g-eg transition of octahedral $\mathrm{Fe}^{2+}$, the $2.5 \mathrm{eV}$ transition to $\mathrm{Fe}^{2+}-\mathrm{Ti}^{4+}$ charge transfer and the $4 \mathrm{eV}$ transition to an $\mathrm{O}-\mathrm{Ti}$ charge transfer. This is consistant with the B3LYP DOS.

Excitation to the $\mathrm{Fe}_{F}^{3+}$ state causes significant shifts in the electronic structure. In HF and B3LYP approximations the Fe $3 d$ states are significantly narrowed and shifted to lower energy (to -18 and $-9 \mathrm{eV}$ in HF and B3LYP, respectively) and the lower edge of the conduction band is of Fe $3 d$ character.

The position of the $\mathrm{O} 2 p$ states is largely unaffected and thus hybridization with these states is reduced dramatically and the upper valence band is dominated by $\mathrm{O} 2 p$ character. In the HF DOS, the Fe $3 d-\mathrm{O} 2 p$ hybridization is reduced to zero leaving a small $\mathrm{Ti} 3 d$ contribution to the almost pure $\mathrm{O}$ $2 p$ character of the valence band. The electron transfered to the Ti site occupies states just below the Fermi energy, about $2 \mathrm{eV}$ above the valence band maximum. This state is reminiscent of the "band gap" state reported in studies of reduced $\mathrm{TiO}_{2} \cdot{ }^{11,34}$

In the B3LYP case the Fermi energy falls in the lower conduction band, occupying some of the $\mathrm{Fe} 3 d \beta$ states resulting in a metallic ground state. The electron on the Ti-site occupies $3 d$ orbitals which hybridise with the $\mathrm{Fe} 3 d$ states and thus contribute both to the metallic states at the Fermi edge and main $\mathrm{Fe} 3 d$ peak at $-9 \mathrm{eV}$.

The above discussion reveals very significant differences in the electronic structure of the $\mathrm{Fe}^{2+} / \mathrm{Ti}^{4+}$ and $\mathrm{Fe}^{3+} / \mathrm{Ti}^{3+}$ states computed at the experimental geometry. It is likely that the nature of the bonding and thus the equilibrium geometry is strongly affected by these differing descriptions; this is explored further below.

\section{B. Geometric structure}

There have been a number of structural determinations of ilmenite. ${ }^{29,33}$ The structural parameters of natural crystal and synthetic crystals are somewhat different and also depend on how the crystals are grown. The structure obtained by Wechsler and Prewitt from a synthetic crystal ${ }^{29}$ is given in Table IV. Morosin et al. ${ }^{33}$ obtained a structure from another synthetic crystal with a slightly different $\mathbf{c}$ parameter (14.04 vs $14.08 \AA$ ). This variation could be due to the formation of hematite $\left(\mathrm{Fe}_{2} \mathrm{O}_{3}\right)$. Natural samples are a solid solution of $\mathrm{Fe}_{2} \mathrm{O}_{3}$ and $\mathrm{FeTiO}_{3}$ and have a c parameter of about $14.04 \AA .35$

In Sec. IV A it was established that the energy difference between the various magnetic states is much smaller than the charge ordering energy scale. Structural optimization has been performed for both charge states within the B3LYP and $\mathrm{HF}$ approximations adopting the ferromagnetic configuration for simplicity.

The structural parameters of the minimum energy geometry computed within the B3LYP and HF approximations are compared to those measured by Wechsler and Prewitt ${ }^{29}$ in Table IV. The structure computed within B3LYP for the $\mathrm{Fe}_{F}^{2+}$ state is in excellent agreement with that observed. The cell vectors and the $c / a$ ratio are accurate to within $1 \%$. The structure predicted for the $\mathrm{Fe}_{F}^{3+}$ underestimates the $c / a$ ratio by over $3 \%$. Similarly structures computed within HF find the cell parameters for the $\mathrm{Fe}_{F}^{2+}$ state in better agreement with experimental results than those of the $\mathrm{Fe}_{F}^{3+}$ state. However the values for the cell vectors computed within HF are slightly larger than those computed within B3LYP, due to the neglect of interelectronic correlation effects in the HF approximation.

Wechsler and Prewitt ${ }^{29}$ deduced the bulk modulus by fitting to a Birch-Murnaghan equation of state ${ }^{28}$ and, constraining the pressure derivative to 4 , obtained a value of $177 \mathrm{GPa}$. Here, if the pressure derivative is constrained to 4 a value of $174 \mathrm{GPa}$ is obtained while an unconstrained fit yields a pressure derivative of 5.1 and a modulus of $172 \mathrm{GPa}$ in good agreement with the observed value.

The structural relaxation alters the relative stability of the different charge states. Within the B3LYP approximation 
TABLE IV. The structure of ilmenite. Experimental values from Wechsler and Prewitt (Ref. 29) and computed values from B3LYP and HF calculations. Values in paretheses indicate percentage error from experiment.

\begin{tabular}{rccccc}
\hline \hline & & \multicolumn{3}{c}{ B3LYP } & \multicolumn{2}{c}{ HF } \\
\cline { 3 - 6 } & Experimental & $\left(\mathrm{Fe}_{F}^{2+}\right)$ & $\left(\mathrm{Fe}_{F}^{3+}\right)$ & $5.165(1.5)$ & $5.223(2.6)$ \\
$a_{0}$ & 5.0875 & $5.150(1.2)$ & $5.183(1.9)$ & $14.200(0.8)$ & $13.695(-2.7)$ \\
$c_{0}$ & 14.0827 & $14.095(0.1)$ & $13.877(-1.5)$ & $2.749(-1.9)$ & $2.622(-5.2)$ \\
$c / a$ & 2.7681 & $2.737(-1.1)$ & $2.677(-3.3)$ & \\
$z(\mathrm{Fe})$ & 0.1446 & 0.1444 & 0.1439 & \\
$z(\mathrm{Ti})$ & 0.3536 & 0.3516 & 0.3507 & \\
$x(\mathrm{O})$ & 0.3172 & 0.2944 & 0.3077 & \\
$y(\mathrm{O})$ & 0.0234 & -0.0251 & 0.0469 & \\
$z(\mathrm{O})$ & 0.2450 & 0.2548 & 0.2498 & \\
$\mathrm{Fe}-\mathrm{O}$ & 2.20 & 2.22 & 2.16 & \\
$\mathrm{Fe}-\mathrm{O}$ & 2.08 & 2.09 & 1.97 & \\
$\mathrm{Ti}-\mathrm{O}$ & 2.09 & 2.09 & 2.11 & \\
$\mathrm{Ti}-\mathrm{O}$ & 1.87 & 1.90 & 2.04 & \\
$\mathrm{~K}_{0}$ & 1.77 & 1.74 & & \\
$\mathrm{~K}^{\prime}$ & 4 & 4 & & \\
\hline \hline
\end{tabular}

there is an increase of the relative stability of the $\mathrm{Fe}_{F}^{3+}$ state by $0.9 \mathrm{eV}$ so that it lies $1.6 \mathrm{eV}$ above the $\mathrm{Fe}_{F}^{2+}$ state. In the $\mathrm{HF}$ approximation the relaxation shift is larger, $1.1 \mathrm{eV}$, and the relaxed $\mathrm{Fe}_{F}^{3+}$ state is predicted to be more stable than the $\mathrm{Fe}_{F}^{2+}$ state by $0.35 \mathrm{eV}$.

\section{DISCUSSION}

In Sec. IV it was established that the computed properties of ilmenite depend strongly on the treatment of exchange and correlation. In particular that in $\mathrm{HF}$ approximation the $\mathrm{Fe}^{3+} / \mathrm{Ti}^{3+}$ state is found to be more stable than the $\mathrm{Fe}^{2+} / \mathrm{Ti}^{4+}$ state. In addition the ionic model was found to be a reasonable basis for the analysis of the charge and spin densities and the electronic structure. This basic picture is achieved in a band structure calculation based on DFT only if the electronic self-interaction is taken into account. An understanding of the role of Coulomb, exchange and correlation interactions can be developed from this basis. In the high-spin states the $\mathrm{Fe}^{3+}$ ion has a $d^{5}$ configuration with five unpaired electrons while the $\mathrm{Fe}^{2+} d 6$ ion has a doubly occupied $d z^{2}$ orbital. The mutual Coulomb repulsion of the electrons in the doubly occupied orbital is apparent in the projected DOS. The correlation energy associated with this electron pair is large and tends to stabilize the $\mathrm{Fe}^{2+}$ ion. The neglect of correlation in the HF approximation therefore leads to an underestimate of the stability of the $\mathrm{Fe}^{2+}$ state. This results in the low value for the charge transfer excitation energy at the experimental geometry and the incorrect prediction of an $\mathrm{Fe}^{3+}$ ground state after structural relaxation. Within the B3LYP approximation the component of exact exchange models a partially screened self interaction and correlation is treated at the GGA level. The correlation energy stablizes the $\mathrm{Fe}^{2+}$ state relative to the $\mathrm{Fe}^{3+}$ state. The excellent agreement between the predicted and measured charge transfer excitation energies indicates that the functional is describing the balance between Coulomb, exchange and correlation interactions rather well. The role of the correlation energy can be isolated by computing it from the self-consistent HF charge density - a so-called a posteriori correction.

The importance of correlation effects in iron systems has also been highlighted in recent studies of $\mathrm{FeS}_{2}$ where it was reported that the poor description of the structure and electronic properties of this material was due to the failure of $\mathrm{HF}$ theory to describe the low-spin $\mathrm{Fe}^{2+}$ ion. ${ }^{36}$ The inclusion of correlation effects, through DFT, hybrid functionals (B3LYP) or a posteriori correlation corrections resulted in much improved structural parameters.

The a posteriori corrections to the HF energies have been computed here using a variety of density functionals. For the relaxed geometries this alters the relative stability of the $\mathrm{Fe}_{F}^{2+}$ and $\mathrm{Fe}_{F}^{3+}$ states. With LDA functionals the shift is about $0.5 \mathrm{eV}$ and so the $\mathrm{Fe}_{F}^{2+}$ is found to be more stable by 0.14-0.21 eV; a smaller shift is found for GGA functionals. This result fully supports the simply ionic picture described above in which the key energy scale is the pair correlation energy of the doubly occupied Fe $d z^{2}$ orbital.

The variation in the electronic structure induced by the charge transfer excitation can also be understood in terms of these energy scales. The shift to lower energies of the Fe- $d$ bands of the $\mathrm{Fe}^{3+}$ state relative to the $\mathrm{Fe}^{2+}$ state is a natural consequence of the reduction in the on-site repulsion and the resulting decrease in hybridization with the $\mathrm{O} 2 p$ bands and the contraction of the $d$ orbitals leads to the narrowing of bands.

The charge tranfer excitation also couples strongly to the geometry. This can be clearly seen in the variation of the $c / a$ ratio. The interatomic distances for the two states are reported in in Table $\mathrm{V}$. 
TABLE V. The interatomic distances (in $\AA$ ) computed with B3LYP. Experimental values from Wechsler and Prewitt (Ref. 29). The $\mathrm{O}-\mathrm{O}$ distances are illustrated in Fig. 6 and lables refer to $(\mathrm{O}-\mathrm{O})_{c \mathrm{FE}}\left[(\mathrm{O}-\mathrm{O})_{c \mathrm{Ti}}\right]$, distance between the O-planes sandwiching $\mathrm{Fe}(\mathrm{Ti})$ bilayers along the $\mathrm{c}$ direction; $(\mathrm{O}-\mathrm{O})_{\mathrm{Fe}-\mathrm{Ti}}, \mathrm{O}-\mathrm{O}$ distances in the face shared by $\mathrm{Fe}-\mathrm{Ti}$ octahedra; $(\mathrm{O}-\mathrm{O})_{\mathrm{Fe}-\text { hole }}\left[(\mathrm{O}-\mathrm{O})_{\mathrm{Ti} \text {-hole }}\right]$, $\mathrm{O}-\mathrm{O}$ distances in the face of an $\mathrm{Fe}(\mathrm{Ti})$ octahedron shared with an octahedral vacancy (hole); $(\mathrm{O}-\mathrm{O})_{\mathrm{Fe}-\mathrm{Fe}}\left[(\mathrm{O}-\mathrm{O})_{\mathrm{Ti}-\mathrm{Ti}}\right], \mathrm{O}-\mathrm{O}$ distance of edges shared by neighboring $\mathrm{Fe}$ (Ti) octahedra; and $(\mathrm{O}-\mathrm{O})_{\mathrm{Fe}}\left[(\mathrm{O}-\mathrm{O})_{\mathrm{Ti}}\right]$, distance of unshared edges in $\mathrm{Fe}(\mathrm{Ti})$ octahedra.

\begin{tabular}{lccc}
\hline \hline & Experiment & Calculated $\left(\mathrm{Fe}_{F}^{2+}\right)$ & Calculated $\left(\mathrm{Fe}_{F}^{3+}\right)$ \\
\hline$(\mathrm{Fe}-\mathrm{O})_{1}$ & 2.078 & 2.099 & 1.974 \\
$(\mathrm{Fe}-\mathrm{O})_{2}$ & 2.201 & 2.222 & 2.160 \\
$\mathrm{Fe}-\mathrm{O}($ mean $)$ & 2.139 & 2.160 & 2.067 \\
$(\mathrm{Ti}-\mathrm{O})_{1}$ & 1.871 & 1.897 & 2.041 \\
$(\mathrm{Ti}-\mathrm{O})_{2}$ & 2.087 & 2.091 & 2.113 \\
$\mathrm{Ti}-\mathrm{O}(\mathrm{mean})$ & 1.981 & 1.994 & 2.077 \\
$\mathrm{mean} M-\mathrm{O}$ & 2.060 & 2.077 & 2.072 \\
$\mathrm{Ti}-\mathrm{Ti}$ & 2.992 & 3.018 & 3.031 \\
$\mathrm{Fe}-\mathrm{Fe}$ & 3.002 & 3.039 & 3.059 \\
$\mathrm{Fe}-\mathrm{Ti}$ & 2.944 & 2.921 & 2.870 \\
$(\mathrm{O}-\mathrm{O})_{c \mathrm{Fe}}$ & 2.487 & 2.485 & 2.307 \\
$(\mathrm{O}-\mathrm{O})_{c \mathrm{Ti}}$ & 2.207 & 2.213 & 2.319 \\
$(\mathrm{O}-\mathrm{O})_{\mathrm{Fe}-\mathrm{Ti}}$ & 2.698 & 2.745 & 2.741 \\
$(\mathrm{O}-\mathrm{O})_{\mathrm{Fe}-\mathrm{hole}}$ & 3.217 & 3.261 & 3.096 \\
$(\mathrm{O}-\mathrm{O})_{\mathrm{Fe}-\mathrm{Fe}}$ & 3.051 & 3.073 & 2.787 \\
$(\mathrm{O}-\mathrm{O})_{\mathrm{Fe}}$ & 3.005 & 3.009 & 2.940 \\
$(\mathrm{O}-\mathrm{O})_{\mathrm{Ti}-\mathrm{hole}}$ & 2.921 & 2.939 & 3.156 \\
$(\mathrm{O}-\mathrm{O})_{\mathrm{Ti}-\mathrm{Ti}}$ & 2.604 & 2.615 & 2.841 \\
$(\mathrm{O}-\mathrm{O})_{\mathrm{Ti}}$ & 2.884 & 2.906 & 2.928 \\
\hline \hline & & & \\
\hline
\end{tabular}

In the $\mathrm{Fe}_{F}^{2+}$ state, the average nearest neighbor $\mathrm{Fe}-\mathrm{O}$ and $\mathrm{Ti}-\mathrm{O}$ bond lengths are 2.16 and $1.99 \AA$, respectively, whereas in the $\mathrm{Fe}_{F}^{3+}$ structure, the mean $\mathrm{Fe}-\mathrm{O}$ and $\mathrm{Ti}-\mathrm{O}$ are 2.07 and $2.08 \AA$, respectively. These variations are consistent with the change in the ionic radii ${ }^{37}$ induced by the charge transfer. The radius of the $\mathrm{Fe}$ ion is reduced from 0.74 to $0.64 \AA$ while the Ti-ion radius increases from 0.68 to about $0.76 \AA$ accounting for the observed changes in average $\mathrm{M}-\mathrm{O}$ distances. Although the $\mathrm{MO}_{6}$ octahedra expand and contract in this way the $\mathrm{Ti}-\mathrm{Ti}$ and $\mathrm{Fe}-\mathrm{Fe}$ distances are almost constant. The Fe-Ti distance is reduced by $0.05 \AA$.

The overall effects of the changes in the ionic radii and the $\mathrm{Fe}-\mathrm{Ti}$ distance are neatly encapsulated in the changes that occur to the separation of the oxygen layers along the $\mathbf{c}$ direction. Two such separations can be defined; the separation of oxygen layers across the Fe bilayer $\left[(\mathrm{O}-\mathrm{O})_{c \mathrm{Fe}}\right]$ and that across the Ti bilayer $\left[(\mathrm{O}-\mathrm{O})_{c \mathrm{Ti}}\right]$. These are illustrated in Fig. 6 and tabulated in Table V. Fe and Ti ions neighbor along $\mathbf{c}$ and one expects the decrease in their separation to correspond to a reduction in the separation of the oxygen planes and an overall reduction in $\mathbf{c}$. The increase in the size of the Ti ions increases $(\mathrm{O}-\mathrm{O})_{c \mathrm{Ti}}$ by $0.11 \AA$ while $(\mathrm{O}-\mathrm{O})_{c \mathrm{Fe}}$ it is reduced by $0.18 \AA$ due to the decrease in the radius of

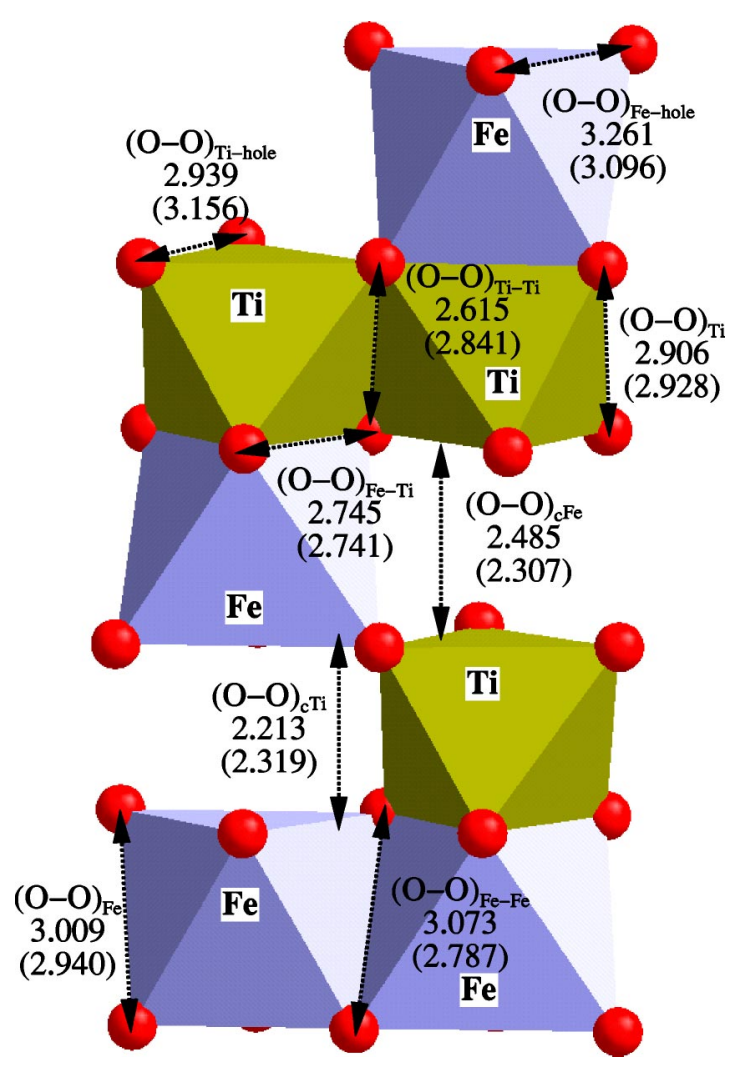

FIG. 6. (Color online) The $\mathrm{O}-\mathrm{O}$ bond distances in the relaxed geometry computed in the B3LYP approximation for the $\mathrm{Fe}_{F}^{2+}$. Values in paratheses are for the $\mathrm{Fe}_{F}^{3+}$ state.

the Fe ions. This leads to an overall decrease in the average separation between oxygen planes of $0.03 \AA$ which is comparable to the decrease in the $\mathrm{Fe}-\mathrm{Ti}$ distance. Since there are six oxygen planes in the crystallographic cell of $\mathrm{FeTiO}_{3}$ the excitation produces an overall contraction of $0.21 \AA$ in the $\mathbf{c}$ parameter.

The $\mathrm{O}-\mathrm{O}$ bond distances within $\mathrm{Fe}$ and $\mathrm{Ti}$ octahedra are also listed in Table V and Fig. 6. Again, the differences in the bond distances between the two charge states can be explained by the changes in the ionic radii. As the $\mathrm{Fe}(\mathrm{Ti})$ ion becomes smaller (bigger) in going from the $2+$ to the $3+(4+$ to $3+)$ state, the $\mathrm{O}-\mathrm{O}$ distances that define the edges of the polyhedron contract (expand). The $(\mathrm{O}-\mathrm{O})_{\mathrm{Fe}-\mathrm{Ti}}$ distance which corresponds to the edges that make up the face that is shared by $\mathrm{Fe}$ and $\mathrm{Ti}$ octahedra remains almost unchanged as the tendency for the Ti octahedron to expand is almost equally matched by the contraction of the Fe octahedron. In the $a b$ plane the layers are not free to expand and contract independently and thus the expansion of the Ti plane leads to an overall increase in the a cell parameter.

\section{CONCLUSIONS}

The ground-state and charge-transfer excitation in ilmenite $\mathrm{FeTiO}_{3}$ are determined by a strong coupling of the geometry with charge, orbital, and spin degrees of freedom. The quantum-mechanical description of these states is very sensitive to the treatment of electronic exchange and corre- 
lation. A hybrid-exchange density functional provides a qualitatively correct description of the ground-state electronic structure. Predicted geometric and elastic parameters are in close agreement with experiment as is the charge transfer excitation energy. The essential features of this functional are its treatment of the electronic self-interaction and its reasonable estimate of the pair correlation energy of the doubly occupied Fe- $d$ orbital. The band gap is predicted to be somewhat lower than that observed but is in much closer correspondence than that computed within an Hartree-Fock or unmodified density functional formalism which results in a metallic ground state.
*Now at Unilever Research and Development, Port Sunlight Laboratory, Merseyside, CH63 3JW, UK.

${ }^{1}$ S. E. Haggerty and V. Sautter, Science 248, 993 (1990).

${ }^{2}$ T. Nagata, S. Uyeda, and S. Akimoto, J. Geomagn. Geoelectr. 4, 22 (1952).

${ }^{3}$ S. Uyeda, J. Geomagn. Geoelectr. 7, 9 (1958).

${ }^{4}$ S. Sunkara and R. K. Pandey, Ceram. Trans. 60, 83 (1995).

${ }^{5}$ D. S. Ginley and M. A. Butler, J. Appl. Phys. 48, 2019 (1977).

${ }^{6}$ D. Sherman, Phys. Chem. Miner. 14, 355 (1987).

${ }^{7}$ K. Leinenweber, W. Utsumi, Y. Tsuchida, T. Yagi, and K. Kurita, Phys. Chem. Miner. 18, 244 (1991).

${ }^{8}$ A. Mehta, K. Leinenweber, A. Navrotsky, and M. Akaogi, Phys. Chem. Miner. 21, 207 (1994).

${ }^{9}$ V. R. Saunders, R. Dovesi, C. Roettie, M. Caus, N. M. Harrison, R. Orlando, and C. M. Zicovich-Wilson, CRYSTAL 98 User's Manual (Universit di Torino, Torino, 1999).

${ }^{10}$ J. Muscat, N. M. Harrison, and G. Thornton, Phys. Rev. B 59, 2320 (1999).

${ }^{11}$ J. Muscat, N. M. Harrison, and G. Thornton, Phys. Rev. B 59, 15457 (1999).

${ }^{12}$ N. M. Harrison, X. G. Wang, J. Muscat, and M. Scheffler, Faraday Discuss. 114, 305 (1999).

${ }^{13}$ W. C. Mackrodt, E. A. Simson, and N. M. Harrison, Surf. Sci. 384, 192 (1997).

${ }^{14}$ J. Muscat, V. Swamy, and N. M. Harrison, Phys. Rev. B 65, 224112 (2002).

${ }^{15}$ M. Catti, G. Valerio, and R. Dovesi, Phys. Rev. B 51, 7441 (1995).

${ }^{16}$ http://www.ch.unito.it/ifm/teorica/crystal.html ("The CRYSTAL basis set library," 2003).

${ }^{17}$ C. Pisani, R. Dovesi, and C. Roetti, Hartree-Fock ab initio Treat- ment of Crystalline Systems (Springer-Verlag, Heidelberg, 1988).

${ }^{18}$ J. D. Pack and H. J. Monkhorst, Phys. Rev. B 16, 1748 (1977).

${ }^{19}$ A. D. Becke, J. Chem. Phys. 98, 5648 (1993).

${ }^{20}$ P. J. Stephens, F. J. Devlin, C. F. Chabalowski, and M. J. Frisch, J. Phys. Chem. 98, 11623 (1994).

${ }^{21}$ A. D. Becke, Phys. Rev. A 38, 3098 (1988).

${ }^{22}$ C. Lee, W. Yang, and R. G. Parr, Phys. Rev. B 37, 785 (1988).

${ }^{23}$ S. H. Vosko, L. Wilk, and M. Nusair, Can. J. Phys. 58, 1200 (1980).

${ }^{24}$ S. Kurth, J. P. Perdew, and P. Blaha, Int. J. Quantum Chem. 75, 889 (1999)

${ }^{25}$ J. Muscat, A. Wander, and N. M. Harrison, Chem. Phys. Lett. 342, 397 (2001).

${ }^{26}$ N. Wilson and J. Muscat, Mol. Simul. 28, 903 (2002).

${ }^{27}$ R. L. Martin and F. Illas, Phys. Rev. Lett. 79, 1539 (1997).

${ }^{28}$ F. D. Murnaghan, Proc. Natl. Acad. Sci. U.S.A. 30, 244 (1944).

${ }^{29}$ B. A. Wechsler and C. T. Prewitt, Am. Mineral. 69, 176 (1984).

${ }^{30}$ H. Kato, Y. Yamaguchi, M. Yamada, S. Funahashi, Y. Nakagawa, and H. Takei, J. Phys. C 19, 6993 (1986).

${ }^{31}$ R. G. J. Strens and B. J. Wood, Miner. Mag. 43, 347 (1979).

${ }^{32}$ M. A. Butler and D. S. Ginley, Chem. Phys. Lett. 47, 319 (1977).

${ }^{33}$ B. Morosin, R. J. Baughman, D. S. Ginley, and M. A. Butler, J. Appl. Crystallogr. 11, 121 (1978).

${ }^{34}$ P. J. D. Lindan, N. M. Harrison, M. J. Gillan, and J. A. White, Phys. Rev. B 55, 15919 (1997).

${ }^{35}$ I. E. Grey (private communication).

${ }^{36}$ J. Muscat, A. Hung, S. Russo, and I. Yarovsky, Phys. Rev. B 65, 054107 (2002).

${ }^{37}$ R. D. Shannon and C. T. Prewitt, Acta Crystallogr., Sect. B: Struct. Crystallogr. Cryst. Chem. 25, 925 (1969). 